\title{
Comparison of Logistics Performance Measurement Tools
}

\author{
D. Strommer ${ }^{1}$, P. Földesi ${ }^{1}$ \\ ${ }^{1}$ Széchenyi István University, Department of Logistics and Forwarding \\ Egyetem tér 1., H-9026 Győr, Hungary \\ e-mail: dianastrommer@gmail.com
}

Abstract: In today's word the role of logistics is getting more important in the operation of enterprises. The competition is big, and cost is one of the most important factors. Logistics is a field which can highly support the reduction of the costs. From the other perspective - the customer satisfaction - logistics also has the role of a supportive function. To get the most out of these two big pillars logistics operation needs to be monitored and measured to give room for further improvement. Currently several methods are available for performance measurement. In this article we present comparison of four of the mainly used performance measurement tools.

Keywords: logistics performance, performance measurement, comparison

\section{Introduction}

This report presents four of the most preferably used performance measurement tools of logistics operations. From the cost perspective logistics is getting more into the focus as this is the area which can be improved and as a result cost reduction can be reached. From the customer's point of view, it is also playing important role in connecting customer and the enterprises and making fruitful cooperation.

The network-oriented development of supply chain led to even bigger complexity. High difficulty is coming from the different goals and perspectives of the different supply chain echelons. The maximalization of personal goals is not working anymore due to the high variety of goods and services which is available for the final customers. Due to the big number of competitors in each level of the chain it is getting complicated to succeed. Reaching customer satisfaction, minimising cost of logistics operation and harmonizing performance of echelons of the chain is crucial. 
There are several measurement tools available to evaluate logistics operations. Each of them has different advantages, aims and focus. There are lot of case studies available, so learning the usage of the tools is not very difficult. The bigger problem is the choice of the alternative fits the most to the goal of enterprise, supply chain or network.

The aim of this paper is not only briefly presenting the four performance measurement systems but also making a comparison between them. The goal is not ranking the tools as both means proper support of performance evaluation process but comparing them based on defined features. This comparison should help choosing the proper tool based on the features defined.

\section{Performance measurement tools}

To evaluate supply chain operation and the member of the chain we need to make measurements. These measures should not be simple as they are evaluating a complex system, but they are highly needed to support strategical decision-making process. There are several tools, methods to use and it is hard to point out which is the best. The performance measurement has wide scale. We can have metrics from a single measurement (such as total cost of the full operation) to complex system which takes into consideration several viewpoints. In today's world the complex measurement is preferred as it is collecting several indicators in a group and tries to evaluate them together. Using a single indicator can easily mislead the evaluation mainly if the targeted area is wider than an enterprise [1].

In the following chapter I will introduce four of the most favourably used performance measurement tools in logistics. All of them is complex tool with several viewpoint which evaluates the operation as a complex process.

\subsection{Balanced Scorecard (BSC)}

The Balanced Scorecard is a frequently used measurement system which is not limited for measuring financial results. The idea was developed by Robert S. Kaplan and David P. Norton in 1992. The tool is mainly supporting the work if the strategical goal is clear and the number of metrics we would like to measure is limited. For structuring the result Balanced Scorecard use four perspectives [7, 8]:

1. Financial perspective

2. Customer perspective

3. Internal business perspective

4. Innovation and growth perspective. 
This model can measure operative processes, but the aim is supporting strategy and long-term changes. The main point in Balanced Scorecard is staying flexible, adaptable with keeping the ability to handle the complexity of measures. The main groups of indicators stated in the model are important in all enterprises and the breakdown of the measurement enables the customization of the system even on enterprise level. Besides the company can also decide regarding the weight of the perspectives based on unique preferences. With regards to the perspectives it is also important to find a way to connect them and point out the parts where they can influence each other $[3,7]$.

Using this method, the two main pillars which was mentioned already can go hand in hand. The cost pressure can be reflected in the financial perspective, but also the customer satisfaction can be taken into consideration. In the model what is more than the already mentioned points are that we can also connect these disciplines to internal business processes and we can see how the development of the internal processes can help with the other factors. What is more, innovation and growth/adaptability can be also integrated into the complex evaluation of the operation, strategy. Basically, the model tries to answer the question 'How does the company succeed?' with the non-financial indicators to support the financial goals and targets [9].

We can differentiate between the perspectives based on the observed period also. Financial perspective shows the financial results of the company, the decisions are made, and the changes occurs due to them. In contrast the other segments mainly focus on the future. What are the areas which can impact future result? How can their improvement help from financial point of view? The limited number of measures also concentrate the focus of decision makers on the focus areas and information not need to be selected, searched out from huge datasets [9].

Having not only financial perspective is a big advantage of the model, but we cannot eliminate the importance of the financial results. In the end that numbers are still the easiest to compare and show the most objectively e.g. the cost of the spare capacity won by the changes [6].

\subsection{Performance Pyramid}

This model has been developed by Lynch and Cross. The model is fitting in in structure of the company hierarchy. The top of the pyramid is matching with the company vision. These goals are supported by the market and financial related indicators from the tactical planning level. In the same level of hierarchy but more into details we can find the elements which have effect on the above-mentioned results such as productivity and customer satisfaction. On the operative level we find the elements that can be influenced the most. These are the followings: quality, 
waste, delivery and cycle time. The below figure shows how the Performance Pyramid is structured [10].

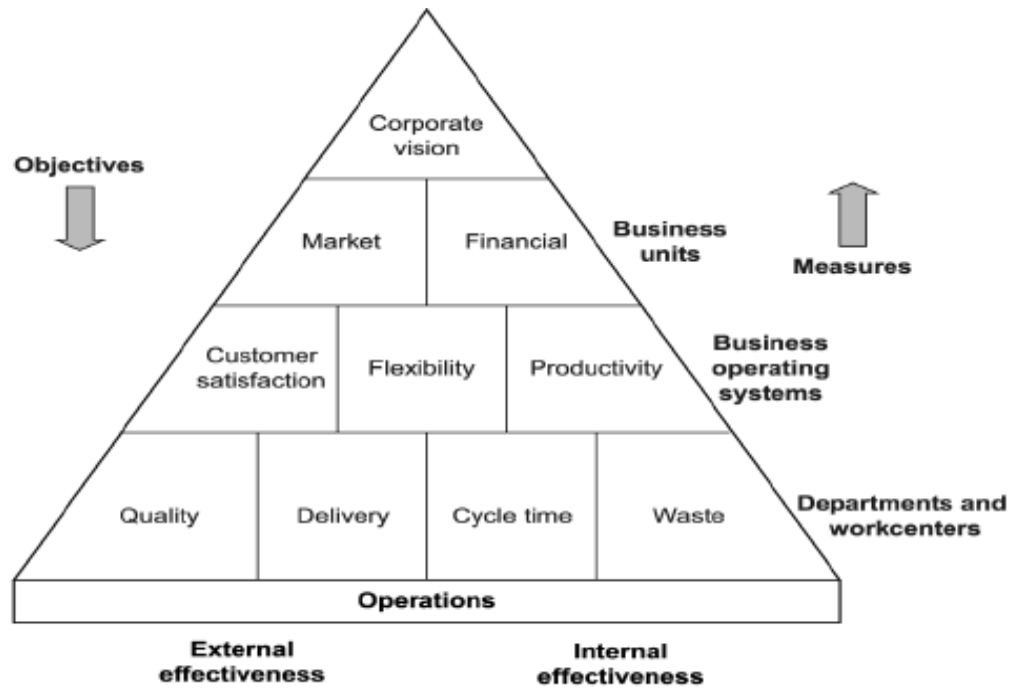

Figure 1.: Performance Pyramid [9]

The core point of the Performance Pyramid is, that instead of top down thinking bottom up method is used. The final goal is placed in the top of the pyramid: corporate vision defined by the company. That makes the basis of lower levels, where the sub-goals are defined based on this. Finally, the steps are initiated in the day-to-day operation. Even though goals are defined strategical level the model is still working in a bottom up way and because of this the goals are defined in all levels. Finally, operative changes turn into strategical goals. This model supports one specific process or problem's evaluation and generation of action plan for it, for continuous improvement it is less helpful. It can be mainly used with traditional, hierarchically shaped companies [9].

\subsection{Tableau de Bord}

This model is a French performance metrics system also called as French Balanced Scorecard. It used as best practice in several French company's operation. The method is concentrating mainly the control of operative processes. The aim is providing overview and control of the company focusing on the future. The goal is not finding deviations for changes but also direct repair of them [4]. 
The definition says that Tableau de Bord is a "tool for the top management of the firm, allowing it a global and quick view of its operations and of the state of its environment" [11]. Initially the tool was used for reporting to initiate conversations on fixed items and hierarchical split of tasks. By the time to adapt to changing requirement the model turned to a performance analyser tool. It has three main elements: objectives, action variables and actions. To reach given objectives (goals) the needed elements should be defined and that is covered by the action variable, which is key factor in reaching the given objective. At the end of the day action plan is generated by the action variables. In this process action variable is key, it need to be controllable and if action variable occurs the probability of the objective following should be high. The selection between the objectives what we want to influence should be based on Pareto's rule, so the most significant improvement area should be selected. In this case there are several objectives (areas to improve) continuously know and always the most relevant is checked.

Tableau de Bord can be used in the different hierarchical levels in a bit different format adapting to the special needs. Traditionally objectives are formed in top level and the responsibility is delegated down to lower levels as more detailed knowledge is needed for the next steps. In the end objectives and action variables are connected. For the action plans defined in the process at least one performance indicator need to be defined. Due to the way responsibility is assigned to the right level cross functional coherence is also helping to reach the goals. As I already mentioned BSC and Tableau de Bord is willingly compared to each other because of the strategic approach broken down to action points. Although they differ for example on the concept and structure [2].

\subsection{Supply Chain Operations Reference (SCOR)}

The model follows process approach aiming to reach effective operation in the supply chain. All activities are checked connected to the material flow and focus on operational efficiency. The model is clearly not following a reporting or analysing approach. It is based on the following steps: plan, source, make, deliver. Plan means the analysis of market information and trends, source stands for the procurement system, make covers the manufacturing, deliver is the process how finished goods reach the customer. It can be completed with and additional element: return, which cover the process of returning goods if needed. It is going beyond a measurement function and aims also to evaluate the issues defined. SCOR model is considering the following attributes: Delivery reliability, flexibility and responsiveness, cost and assets. The focus is on logistical flow and the echelons participating on it. Finance can be part of the measurement, but it will never be in the in the focus area. 
The below figure shows that connected SCOR evaluations can give feedback on the operation of the chain. The model can be used in each element but for real improvement the interacting echelons cannot be handled separately [9, 12].

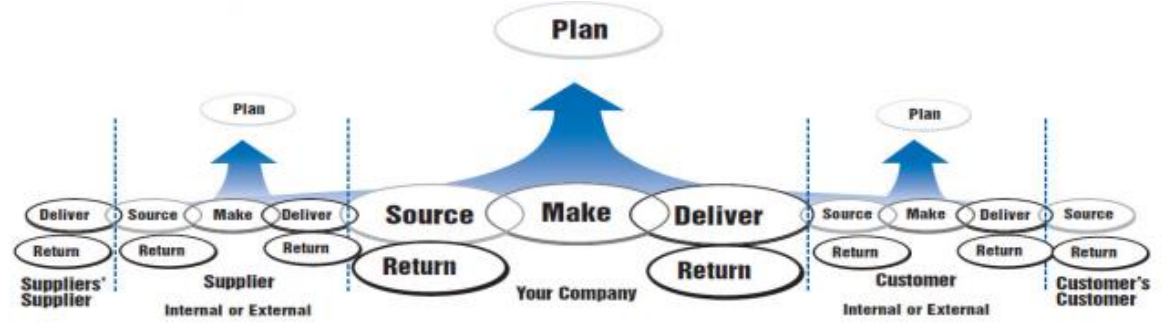

Figure 2.: SCOR model [5]

The model has four levels regarding the implementation. The first level defines the main supply chain processes, support the SCM objectives. The second level goes more into details, explain main process categories. The third level of breakdown consist further benchmarks, information, explanation on processes and capabilities. Finally, the fourth level stands for the implementation [5].

\section{Comparison}

In this report I summarised the basic information about four performance measurement system. Each of them is widely used in the industry. All of them has advantages and disadvantages. In the following chapter I compare the methods presented before based on the following features:

- $\quad$ key indicators. Are the key indicators defined in the given metrics?

- $\quad$ perspectives: Are the model defines different view-points, perspectives or categories of measures?

- non-financial: Are the measured parts beyond financial attributes?

- strategy: what strategy is the model following? bottom up or top down?

- network: is the model designed to measure a company? or capable of measuring a network or chain?

- operations: is the analytics broken down to operational level?

- orientation: customer or company point of view is followed? process or result oriented measures are used? 
- management tool: is the measurement designed partly or fully to support strategical decision-making process?

- reporting: is the aim of the model fully or partially report about the status of the performance?

The comparison is made through different angles. Each of them is important because they are highly differentiated between the companies who use it. The below table shows how the introduced methods can be evaluated based on them.

Table 1. Comparison of performance measurements

\begin{tabular}{|c|c|c|c|c|}
\hline Features & BSC & $\begin{array}{c}\text { Performance } \\
\text { Pyramid }\end{array}$ & $\begin{array}{c}\text { Tableau de } \\
\text { Bord }\end{array}$ & SCOR \\
\hline key indicators & yes & no & yes & yes \\
\hline perspectives & yes & no & no & yes \\
\hline non-financial & yes & yes & yes & yes \\
\hline strategy & top down & bottom up & top down & top down \\
\hline network & yes & no & yes & yes \\
\hline operations & yes & yes & yes & yes \\
\hline orientation & both & process & both & process \\
\hline management tool & yes & no & yes & no \\
\hline reporting & yes & no & yes & no \\
\hline
\end{tabular}

\subsection{Methods based on the defined features}

Balanced Scorecard is a management tool set up for support strategical goals, decision making of the company. The frame of the model is based on four perspectives which define the structure and ensure that non-financial measures can also take part in the examination, group all the relevant indicators. The action points, next steps are broken down to operative level, but the decision is coming from higher hierarchical point of view. The BSC can be used for a single echelon of the supply chain or for cooperation or network of companies, it depends on the finally defined indicators under the different perspectives. The view-point is also quite flexible, defined by the indicators. It can be both process and result oriented, it is also mainly depending on how the weights of the perspectives set and what kind of indicators are defined during the measurement.

Performance Pyramid is providing company specific support, not useable for networks. The focus is on the development of the operational processes or elimination of a problem with a bottom up strategy. Goals are not predefined, they are set based on the operative level. Instruction, frame or set of indicators are not given. The model is more a process improvement method than a set of metrics. It is 
based on hierarchy levels. The method is beyond financial measures. The method is not meant to be a strategical decision-making tool but an operational improvement system. Objectives are existing from the top down communication, but the focus is on the bottom up development.

Tableau de Bord as it was mentioned is very similar to the Balanced Scorecard from several extent. It is also a tool supporting strategical decision making and in the same time detailed system with elements broken down to operational level. As it is also defining objectives based on the strategy the strategical flow here also works in the top down way. The objectives mentioned are the key indicators which are chosen to be improved based on the impact for the future, but they are not grouped into categories or put in any frame. Objectives can be financial but do not have to be so this model is also beyond purely financial measures. The method can be easily extended to chain or network of echelons, or it can be company specific. Depending on the scope of the model the action variables and action plans will be different. Regarding orientation the number of possibilities is not limited also. It can be process or problem orientated depending on the content of the objectives and action variables.

The SCOR model is also supporting the strategical decision making but the main goal is not that but gaining advantages of operational changes in the material flow. It is process orientated, so the measures are set to evaluate and improve the processes in place. The direction of indicators is coming in a top down stream of communication. The usage is optimal more for networks than for echelons of the supply chain. The goals are set based on the company vision but the breakdown for tactical and operative level is also part of the methodology. The focus of the measurement is process improvement from different view-points, so it is also beyond the financial indicators. The target of this model is resulting better efficiency in the material flow. It is more of a non-financial approach. The indicators can be grouped in the model-based levels (following the hierarchy) or based on the flow of material in the supply chain (plan, source, make, delivery).

\subsection{Differences based on the defined features}

One of the main requirement towards the performance measurement tools is being able to handle complexity and support efficiency. This is true for all the examined methods and this is one of the elements what makes them widely used. As among the requirements stated by the users there are no two equals also among the methods there will be no uniform content that makes them adaptable for various scenarios.

Another basic requirement is going beyond the financial metrics and gain better efficiency. This is also part of all the checked methods. They can handle well non- 
financial metrics. That helps the growth of efficiency and effectiveness of operations.

Among the four tools Performance Pyramid differs the most. All other examined tools are based on key indicators, shaped more in a top down way of thinking. Performance Pyramid aims to evaluate a process or a company it is not willing to handle any wider range.

The SCOR model also a bit differs from all others as it is aiming to execute changes not on one echelon but in a chain or network of companies. Most of the other models are also capable of this but the main structure is not originally designed for this.

Regarding key indicators, measurements in three of the models we can find instructions. In two of these cases the indicators are integrated in a structured way. This structure can help during the understanding or analysis of results or even during shaping other indicators to measure. It can be also used during the decision-making process or reporting of the results. It is also visible that this frame is missing in Performance Pyramid, which is the only tool among the four which is the less compatible with complexity. We can state that if not only processes but also relations and cooperative actions need to be examined than structure of measures can be supportive.

As the initiatives are coming mainly connected to the company's strategy it is not a big surprise that most of the models are working in a top down way. They are fed buy the vision of the company. If we only take this into consideration it may seems that the measures are only for supporting presentation of strategical goals. But the measures are delegated and broken down to operative level. With this at the end of the day top down visions relate to practical steps.

It is also important from what perspective we would like to see the effectiveness of the operation. If we want to have minimum cost and best usage of capacity the focus is in processes. It can be realised for example through setting low stock level or even avoid safety stock. With this no money would stand in stock but it would hardly satisfy the customers need due to the long lead time. It can be managed the other way around as well. That would mean in the given example: safety is set based on agreement with customer or historical sales information. None of the perspectives is good or bad. Focus is question of decision. In this example if the product is hardly replaceable there is no need for the customer or result oriented thinking. In case of e.g. FMCG products it is necessary to start analysis of processes from the required results point.

As table above clearly show SCOR model and Performance Pyramid is set up for process-oriented metrics. It brings the importance of processes and chain or 
connection of them. While the other two models can be set up from both perspectives. It mainly depends on the key indicators which are phrased in the model. In case on Balanced Scorecard it also depends on the weight we give to each perspective, so we can differentiate.

Both BSC and Tableau de Bord are management tools. That mean that the result of the analysis is preferably used for the decision-making process later. Due to this the models have supportive functionality. In contrast SCOR is aiming to implement changes right away if there is any possibility for them. This does not mean that BSC and Tableau de Bord is not supporting the implementation but means that they have a functionality of management support.

We cannot say that any of the introduced systems are fully dedicated to reporting purpose. This is only a subsidiary function. Two of the examined cases is not created to support any kind of reporting functionality. As it was already mentioned SCOR is a practice-based tool which is highly focusing on the implementation and practice. Performance Pyramid with the bottom up approach is also not a reporting tool.

\subsection{Comparison of Tableau de Bord and Balanced Scorecard}

As the similarity between the Balanced Scorecard and Tableau de Bord is quite big it also worth to compare only the two them. Both two tools are a top down strategy based supportive methods which aim to examine the operative processes based on the company vision and strategical goals. The measures are not made on high level but translated to day-to-day tasks and with this the vision is directly connected with employee actions.

We can also state that the bypass of financial dominance in measures is aim of each. Both model offers several key indicators what can be used in the frame of the measurement. But it is also recommended in all cases to keep the focus with weights or prioritized KPIs. This ensure that the user would not lose the real results between the huge number of metrics. The mentioned frame is usefully mainly due to the decision-making support functionality of the models and due to the reporting aims.

Although the similarity between the methods are indisputable we still can see some differences. The biggest gap between the model is in the level of predetermination. Balanced Scorecard has four perspectives which strictly determine how the result will look like and partially also determine what measures can be introduced in the model. From this perspective Tableau de Bord is designed freer, not connected tightly with a predesigned structure. On one hand this makes Balanced Scorecard easier to understand and set up but on the other hand it can be hindering factor from the adaptability point of view. 
Other difference can be find if the focus or result of the model is examined. In case of Tableau de Bord the aim is defining an objective and through an action plan reach this goal which drives to development. In contrast Balanced Scorecard tries to make measurable, quantitative performance indicators and the focus is more on the result itself than on the development.

\section{Conclusion}

As the changes of requirements are more and more pushing towards cost reduction the effectiveness of the companies and logistics operation is remaining in focus. Performance measurement tools are supporting this changes and challenges. During the past years they changed according to the modified market needs. In this report four measurement has been introduced. Each method has its own advantages and disadvantages, each of them was created to ensure the effective operation of the company.

This report's aim to give a starting point of selection between the four examined methods based on the user specific requirements. Table 1. summaries the comparison of the different measures. It is clearly visible that in case of network evaluation we cannot use Performance Pyramid, but it is totally fitting with a hierarchical setup. It is also definite that SCOR model is the most practice oriented and focus most on network-based efficiency. Regarding Balanced Scorecard and Tableau de Bord two very similar tool has been introduced with determined structure and defined key indicators. The setting of order is not targeted in this report. Each tool is appropriate in its own field. One of the core tasks is defining the main features of the model we are searching for, so it can support further goals better.

\section{References}

[1] N. Asadi, Performance Indicators in Internal Logistic systems, in 2012 International Conference on Innovation and Information Management (ICIIM 2012) IPCSIT vol. 36 (2012) (C) (2012) IACSIT Press, Singapore, Singapore, 2012, pp. 48-52.

URL http://www.ipcsit.com/vol36/010-ICIIM2012-M0026.pdf

[2] A. Bourguignon, V. Malleret, H. Nørreklit, The American balanced scorecard versus the French tableau de bord: the ideological dimension, Management Accounting Research 15 (2) (2004) 107-134. https://doi.org/10.1016/j.mar.2003.12.006 
[3] P. Chytas, M. Glykas, G. Valiris, A proactive balanced scorecard, International Journal of Information Management 31 (5) (2011) pp. 460-468. https://doi.org/10.1016/j.ijinfomgt.2010.12.007

[4] J. H. Daum, French Tableau de Bord: Better than the Balanced Scorecard?, Der Controlling Berater 7 (2005) pp. 459-502.

[5] G. E. Delipinar, B. Kocaoglu, Using SCOR model to gain competitive advantage: A Literature review, Procedia - Social and Behavioral Sciences 229 pp. 398-406. https://doi.org/10.1016/j.sbspro.2016.07.150

[6] M. J. Epstein, J. F. Manzoni, The Balanced Scorecard and Tableau de Bord: Global Perspective on Translating Strategy into Action, Management Accounting: Official Magazine of Institute of Management Accountants 79 (2) (1997) pp. 28-36.

URL https://flora.insead.edu/fichiersti_wp/inseadwp1997/97-82.pdf

[7] Z. T. Kalender, Ö. Vayvay, The Fifth Pillar of the Balanced Scorecard: Sustainability, Procedia - Social and Behavioral Sciences 235 pp. 76-83. https://doi.org/10.1016/j.sbspro.2016.11.027

[8] R. S. Kaplan, D. P. Norton, The Balanced Scorecard - Measures that Drive Performance, Harvard Business Review January-February (1992) pp. 71-79.

[9] G. P. Kurien, M. N. Qureshi, Study of performance measurement practices in supply chain management, International Journal of Business, Management and Social Sciences 2 (4) (2011) pp. 19-34.

[10] R. L. Lynch, K. F. Cross, Measure Up! Yardsticks for continuous improvement, 2nd Edition, Wiley, Cambridge, 1995.

[11] J.-L. Malo, Les tableaux de bord comme signe d'une gestion et d'une comptabilité, in: Melanges en l'honneur du professeur Claude Pérochon, Foucher, Paris, 1995, pp. 357-376, in French.

[12] J. Thakkar, A. Kanda, S. Deshmukh, Supply chain performance measurement framework for small and medium scale enterprises, Benchmarking: An International Journal 16 (5) (2009) pp. 702-723. https://doi.org/10.1108/14635770910987878 